\title{
Effect of wavelength of fish-like undulation of a hydrofoil in a free-stream flow
}

\author{
THEKKETHIL NAMSHAD, MUKUL SHRIVASTAVA, AMIT AGRAWAL \\ and ATUL SHARMA*D
}

Department of Mechanical Engineering, Indian Institute of Technology Bombay, Mumbai 400076, India

e-mail: atulsharma@iitb.ac.in

MS received 27 May 2016; revised 11 October 2016; accepted 20 October 2016

\begin{abstract}
Fish-like undulating body was proposed as an efficient propulsion system, and various mechanisms of thrust generation in this type of propulsion are found in the literature-separately for undulating and pitching fishes/foil. The present work proposes a unified study for undulating and pitching foil, by varying wavelength $\lambda$ (from 0.8 to 8.0 ) of a wave travelling backwards over the NACA0012 hydrofoil in a free-stream flow; the larger wavelength is shown to lead to the transition from the undulating motion to pitching motion. The effect of wavelength of undulation is studied numerically at a Reynolds number $R e=4000$, maximum amplitude of undulation $A_{\max }=0.1$ and non-dimensional frequency of undulation $S t=0.4$, using level-set immersedboundary-method based in-house 2D code. The Navier-Stokes equation governing the fluid flow is solved using a fully implicit finite-volume method, while level-set equation governing the movement of the hydrofoil is solved using an explicit finite-difference method. It is presented here that the thrust generation mechanism for the low wavelength case undulating $(\lambda=0.8)$ foil is different from the mechanism for the high wavelength pitching foil. With increasing wavelength, mean thrust coefficient of the undulating foil increases and asymptotes to value for the pure pitching foil. Furthermore, the ratio of maximum thrust coefficient to maximum lateral force coefficient is found to be larger for the smaller wavelength undulating foil as compared with the larger wavelength pitching foil.
\end{abstract}

Keywords. Fish-like locomotion; pitching; undulation; wavelength.

\section{Introduction}

Free-stream flow across a cylinder is a classical fluid dynamics problem; however, most of the studies are reported for stationary as compared with moving cylinder. In general, the moving cylinder problem corresponds to the interaction of a moving (rigid or deformable) structure within a stationary or moving fluid, called as a FluidStructure interaction (FSI) problem. Due to a large number of upcoming engineering applications, there is a large body of work on the coupled fluid and solid dynamics problem in the last decade. Most of the engineering applications correspond to a periodic FSI such that there is a periodic motion of the structure. One such nature-inspired FSI considered here is the hydrodynamics across a fish-like undulating hydrofoil. A better understanding of the thrust generation mechanism used by the aquatic animals can help in discovering new and efficient propulsion systems. The mimicking of such natural systems led to the evolution of a subject called as biomimetics. However, it is a great

*For correspondence challenge to come up with an engineering system that can mimic the flexibility as well as the motion of natural systems. This is true for an experimental but not for a numerical work, especially while mimicking the motion. Thus, an opportunity exists for Computational Fluid Dynamics (CFD) to bear on these experimentally challenging problems.

There are numerous studies, more by zoologists as compared with fluid fluid-dynamicists, on the hydrodynamics and propulsion during the periodic swimming movements of the fishes. Breder [1] proposed that the periodic motions are generated by two major categories in almost all the fishes: BCF (Body and/or Caudal Fin) and MPF (Median and/or Paired Fin) motion. For both BCF and MPF propulsion, a further classification is based on the characteristics of the periodic motion: undulating motion involves the passage of a wave along the propulsive structure, while in the pitching motion the propulsive structure swivels on its base without exhibiting a wave formation [2]. The laterally undulating and the pitching motion of a 2D hydrofoil are shown as the top view of a swimming fish in figure 1 , considering the same chord 
(a)

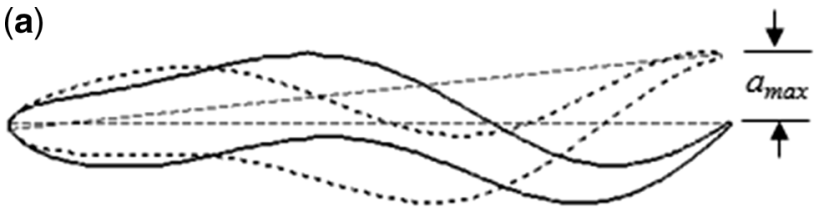

(b)

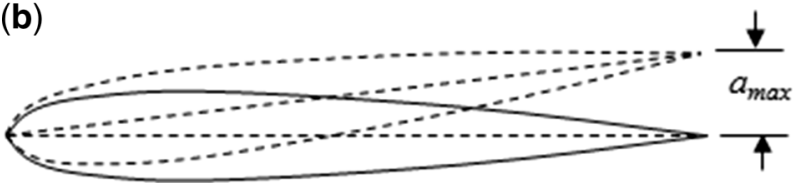

Figure 1. (a) Undulating and (b) pitching motion of the hydrofoil.

length $c$ and amplitude $a_{\max }$ for both these types of periodic motion. The undulating motion is imposed on the pitching hydrofoil by a backwards travelling wave of wavelength $\lambda$.

The BCF as compared with MPF-motion-based propulsion is found in the majority of fishes [2], categorized further by Breder [1] into four different types of swimming modes: anguilliform, sub-carangiform, carangiform and thunniform. The periodic motion corresponds to almost whole body for the anguilliform fishes (such as eel and lamprey), and almost restricted to the caudal fin (in the posterior part) for the thunniform fishes (such as shark, tuna and marine mammals); however, for the sub-carangiform and carangiform types, both body motion and caudal-fin motion are of comparable magnitude. However, note that the motion changes from undulatory motion (of almost whole body) in anguilliform to pitching and heaving motion (of only caudal fin) in thunniform types of fishes [2]. Also, note that there is no caudal fin for most of the anguilliform fishes and the size of the body is the biggest for the thunniform fishes. The propulsion of thunniform fishes is mainly due to a lateral pitching of the caudal fin, with and without heaving motion of the airfoil-shaped cross-section [3]. The motion of the 2D cross-section of the caudal-fin corresponds to the pitching motion of a $2 \mathrm{D}$ hydrofoil; however, if the body is also undergoing lateral oscillation, the motion of the 2D hydrofoil corresponds to both pitching motion and heaving motion.

For free-stream flow across an undulating fish-like body, various studies in the published literature are presented in this paragraph. This was probably initiated by Lighthill [4], using inviscid theory and slender body approximations, to predict the thrust generation mechanism and the maximum efficiency condition (the wave velocity near the free-stream velocity). Liu et al [5] presented a 2D numerical study considering flow over a tadpole-like body, and obtained the conditions for maximum and minimum thrust generation. The maximum efficiency condition was obtained when the body takes "C" shape in which the area to push forward is maximum. Muller et al [6] experimentally studied the thrust generation in anguilliform fish. They found that thrust is generated all over the body by continuous suction and pressure flows generated by the undulating motion. Body vortex formation was found along the body due to the pressure and suction flows [7]. Jian et al [8] carried out a numerical investigation on flow across an undulating NACA0012 hydrofoil, and found that there exists a critical speed of the undulating wave above which thrust generation occurs. A numerical study conducted by Kern and Koumoutsakos [9] on flow over an anguilliform fish-like body compared the flow fields for 2D and 3D cases. They found that the three dimensionality of the flow is responsible for the instability of shear layer and it leads to the formation of secondary vortices. The $2 \mathrm{D}$ simulations were found to be efficient in predicting the propulsive parameters with minimum error. Thrust generation mechanisms in anguilliform and carangiform type of fish-like bodies were analysed by Borazjani and Sotiropoulos [10-12]. They studied the effect of body shape and kinematics of undulation on the propulsive performance of both types of bodies.

For hydrodynamics across only pitching or both pitching and heaving hydrofoil, various studies from the published literature are presented in this paragraph. For the free-stream flow over pitching NACA0012 airfoil, Koochesfahani [13] experimentally investigated the effects of amplitude, frequency and shape of the waveform on propulsive performance. He found that the axial flow in the cores of the wake increases with increasing pitching amplitude. Furthermore, this problem was studied numerically by Pedro et al [14], with a demonstration of formation of reverse von Karman vortex street and a discussion on thrust generation mechanism. Shinde and Arakeri [15] experimentally studied the hydrodynamics of pitching NACA0015 airfoil in a quiescent fluid (Strouhal number of infinity). Triantafyllou et al [16] performed an experimental study on pitching and heaving hydrofoil and demonstrated vorticity control and a jet flow behind the foil, which results in thrust generation. They found unstable wave formation behind the foil, which rolls up and forms reverse von Karman vortices for the Strouhal number above a certain range. They reported that the maximum efficiency occurs for the Strouhal number between 0.25 and 0.35 . This problem was also experimentally studied by Anderson et al [17], for the conditions resulting in strong and efficient reverse von Karman vortices. Gopalkrishnan et al [18] conducted an experimental study on free-stream flow over a pitching and heaving NACA0012 hydrofoil behind a D-section cylinder. They studied the interaction of vortices formed from the cylinder and the hydrofoil and concluded that the phase difference between these vortices plays an important role in determining the propulsive performance. Guglielmini and Blondeaux [19] numerically studied the hydrodynamics of flow over a pitching and heaving elliptical foil, and concluded that the heaving motion is preferred when the fluid motion is present while pitching motion is suggested in still fluid. 
The conclusion drawn from the above literature survey is that there are numerous 2D studies on free-stream flow across either only pitching or both pitching and undulating hydrofoil [4-19]. The two types of motion for the two types of fishes, pitching fin for thunniform and body undulation for anguilliform fishes, lead to different mechanisms of thrust generation, and their combination is encountered in the sub-carangiform and carangiform type of fishes. Sfakiotakis et al [2] mentioned that the two types of motion should be considered as a continuum, since oscillatory movements can eventually be derived from the gradual increase of the undulation wavelength. However, a single governing-parameter-based combined study for the two types of fish-like locomotion is not found in the literaturedone in the present work. Thus, the novelty of the present work is a unified study considering both the types of motion together, by considering the effect of undulation wavelength whose larger values convert the undulatory motion to the pitching motion. The present study is motivated by the fact that the two types of motion/mechanism can be used in an optimum way so as to obtain the maximum efficiency. This will lead to a novel and efficient propulsion system, based on fish-like locomotion. The hydrodynamics over the fish body is $3 \mathrm{D}$, with the two types of motion in the same plane and over the different parts of the BCF types of fishes - the lateral undulatory motion of the body and the lateral pitching motion of the caudal fin. However, to limit the present study to $2 \mathrm{D}$, both types of motion are considered for the same hydrofoil, considered here as NACA0012.

\section{Physical description of the problem}

The present problem on free-stream flow across an undulating NACA0012 hydrofoil is shown in figure 2. The figure shows $u_{\infty}$ as the free-stream velocity and $c$ as the chord length of the hydrofoil, considered as the velocity and length scale for the present non-dimensional study, respectively. The figure corresponds to the top view of the fish-like body undulating in the lateral direction; the left

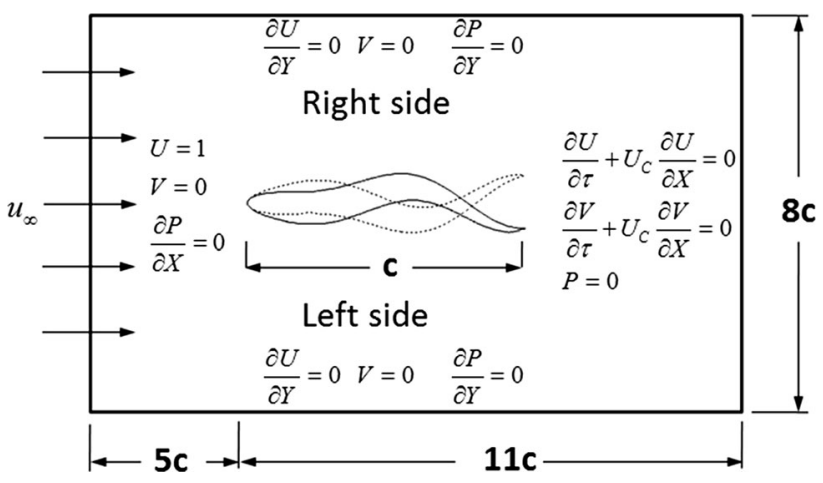

Figure 2. Computational domain for free-stream flow over an undulating NACA0012 hydrofoi. and right sides shown in the figure are the lateral directions. The figure shows the computational domain (with dimensions in terms of non-dimensional units) and the boundary conditions (BCs) considered in the present simulations. The $\mathrm{BCs}$ correspond to the free-slip $\mathrm{BC}$ at the lateral (left and right) boundary. A uniform velocity is set at the inlet boundary and a convective outflow $\mathrm{BC}$ is used at the outlet boundary; the convective velocity $U_{C}$ is set equal to the non-dimensional average streamwise velocity of 1.0 [20]. Note that the undulating motion is used to model the body motion for the fish-like propulsion, which changes to the pitching motion at larger wavelength of backwards travelling wave $\lambda$, used as a parameter to prescribe the undulation (presented later). The pitching motion is used to model the caudal-fin/tail motion for the fish-like propulsion.

An initial shape of the hydrofoil at the onset of fish-like locomotion is shown in figure 3 , mimicking the wavy shape of the body of fish. The wavy hydrofoil is obtained from the straight hydrofoil by prescribing a non-dimensional lateral displacement $\triangle Y$ that varies along the length of the body as

$$
\triangle Y=A(X) \sin \left(2 \pi \frac{X}{\lambda}\right)
$$

where $X$ is the non-dimensional distance from the head and $\lambda$ is the non-dimensional wavelength of a wave travelling across the hydrofoil. Furthermore, $A(X)$ is the chordwise flexure amplitude of undulation, modelled here by a static head linear motion (SHLM) type of kinematics as

$$
A(X)=A_{\max } X
$$

where $A_{\max }$ is the maximum non-dimensional lateral displacement, called as the amplitude of undulation. For modelling the periodic undulatory motion, Eq. (1) is given a time-wise variation.

$$
\text { Undulating motion: } \triangle Y=A(X) \sin \left[2 \pi\left(\frac{X}{\lambda}-\frac{S t \tau}{2 A_{\max }}\right)\right]
$$

where $\tau$ is the non-dimensional time $\left(\equiv t u_{\infty} / c\right)$ and $S t\left(\equiv 2 a_{\max } f / u_{\infty}\right)$ is the Strouhal number (non-dimensional frequency of undulation $f$ ). When the wavelength of undulation is very large, $\lambda \rightarrow \infty$, the above equation for the

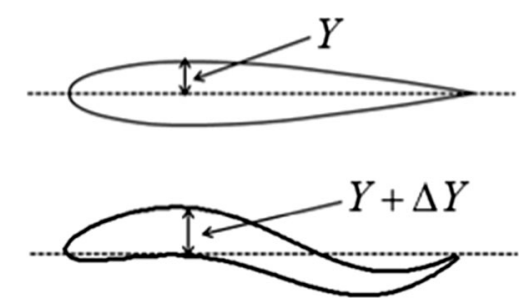

Figure 3. Streamwise varying lateral displacement $\Delta Y$ of NACA0012 hydrofoil to obtain its initial wavy/fish-like shape, at the onset of locomotion. 
undulating motion corresponds to the pitching motion about the horizontal centre line.

$$
\text { Pitching motion: } \triangle Y=A(X) \sin \left(-2 \pi \frac{S t \tau}{2 A_{\max }}\right) \text {. }
$$

\section{Numerical description}

The moving boundary problem of the unified pitching and undulating motion is solved using level-set-based immersed boundary method, proposed by Shrivastava et al [21]. The hydrodynamics of the flow field is governed by the NavierStokes(NS) equations, while the solid-fluid interface is tracked using level-set(LS) equations.

Navier-Stokes equations

Continuity:

$$
\nabla \cdot \vec{U}=0
$$

Momentum: $\quad \frac{\partial \vec{U}}{\partial \tau}+\nabla \cdot(\vec{U} \vec{U})=-\nabla P+\frac{1}{R e} \nabla^{2} \vec{U}$

Level set equations

$$
\begin{array}{cc}
\text { Advection: } & \frac{\partial \phi}{\partial \tau}+\vec{U}_{a d v} . \nabla \phi=0 \\
\text { Reinitialization: } & \frac{\partial \phi}{\partial \tau_{s}}+S_{\varepsilon}(|\nabla \phi|-1)=0
\end{array}
$$

where $\vec{U}$ is the non-dimensional velocity of the fluid, $P\left(\equiv p / 0.5 \rho u_{\infty}^{2}\right)$ is the non-dimensional pressure and $\vec{U}_{a d v}$ is the advecting velocity of the solid-fluid interface. The Reynolds number $R e$ is defined based on the chord length of the hydrofoil $\left(R e=\rho u_{\infty} c / \mu\right)$. The LS function $\phi$ is defined as the normal distance function which is positive in the fluid and negative in the solid. For the re-initialization equation, $\tau_{s}$ is the pseudo time and $S_{\varepsilon}\left(\phi_{0}\right)$ is the smoothened sign function.

For the present work, an in-house code developed by Shrivastava et al [21] is used. The code is based on the finite-volume method for solving the NS equations, and finite-difference method for the LS equations. The LS function $\phi$ is a normal distance function which is positive in the fluid region and negative in the solid region. The solid-fluid boundary is identified using the LS function. At each time step, LS equations are solved using 3rd order Runge-Kutta method for the temporal-term, and 5th order WENO (weighted essentially non-oscillatory scheme) for the spatial-terms in order to compute the LS function. The NS equations are solved in the fluid region $(\phi$ is $+v e)$ using a fully implicit pressure correction method on a co-located Cartesian grid system. The border grid points in the fluid (which have at least one neighbouring cell in the solid) are treated separately. The face velocity for border grid points is taken as the velocity of the solid neighbouring grid point.
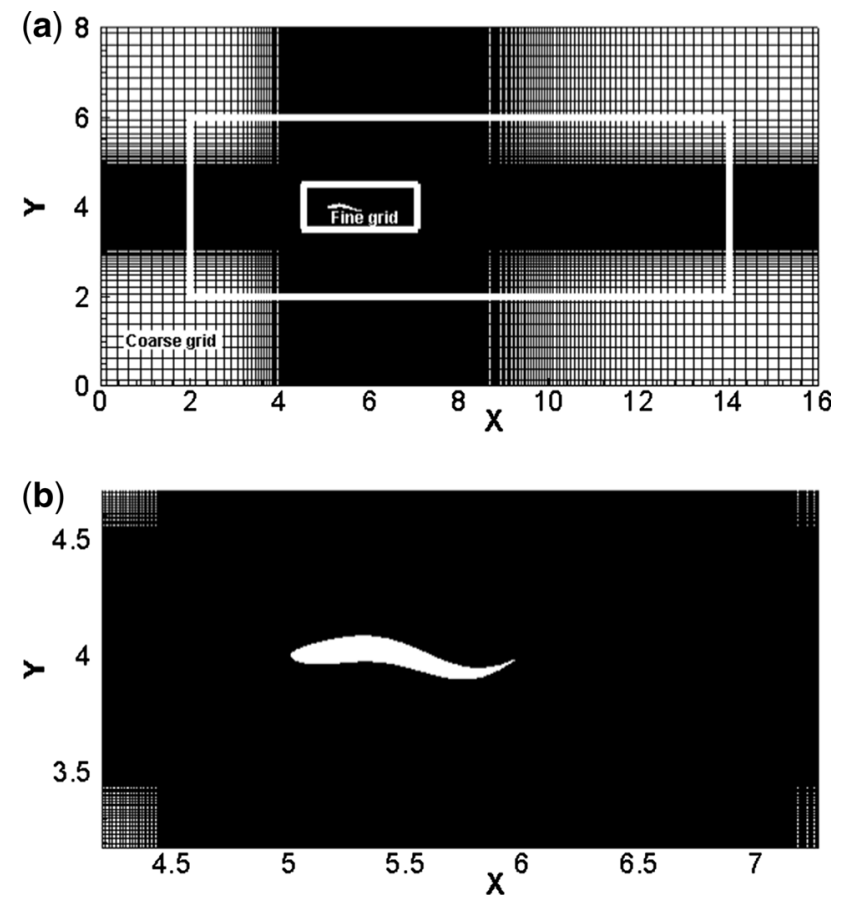

Figure 4. (a) Grid employed for the simulation of flow over undulating hydrofoil and (b) zoomed view of the grid near the hydrofoil.

A domain-length convergence study is done at $R e=4000, S t=0.4, \lambda=0.8$, and $A_{\max }=0.1$, for three different non-dimensional domain sizes $14 \times 8,16 \times 8$ and $20 \times 8$; downstream domain length in figure 2 is varied as 9,11 and 15. For a domain length as compared with the largest length, the percentage difference is $0.26 \%$ for $C_{T m}$ and $0.69 \%$ for $C_{\text {Lrms }}$ on a domain size of $14 \times 8$, and reduces to $0.1 \%$ for $C_{T m}$ and $0.24 \%$ for $C_{L r m s}$ on a domain size of $16 \times 8$. Thus, a domain size of $16 \times 8$ is used is considered sufficient for the domain-length-independent results, and is used in the present study. A non-uniform grid size of $768 \times 314$ is used in the present work, shown in figure 4. The grid structure is such that a uniform coarsest grid size of 0.25 is used two non-dimensional units upstream, downstream, above and below the foil, while a finest grid size of $\delta=0.005$ is used in the region (of size $2.5 \times 1$ ) near the body. In the region in-between the fine and coarse grids, the grid stretches from 0.005 to 0.25 . The grid size is arrived after a grid-independent study at $R e=4000, S t=0.4, \lambda=0.8$ and $A_{\max }=0.1$, with three grid sizes, $502 \times 250,768 \times 314$, and $1072 \times 448$, with the finest grid sizes as $0.0075,0.005$, and 0.003 , respectively. For a grid size as compared with the finest grid, the percentage difference is $0.34 \%$ for $C_{T m}$ and $0.80 \%$ for $C_{\text {Lrms }}$ on a grid size of $502 \times 250$, and reduces to $0.15 \%$ for $C_{T m}$ and $0.27 \%$ for $C_{L r m s}$ on a grid size of $768 \times 314$. Thus, a grid size of $768 \times 314$ is considered sufficient for the grid-independent results, and is used in the present work. 

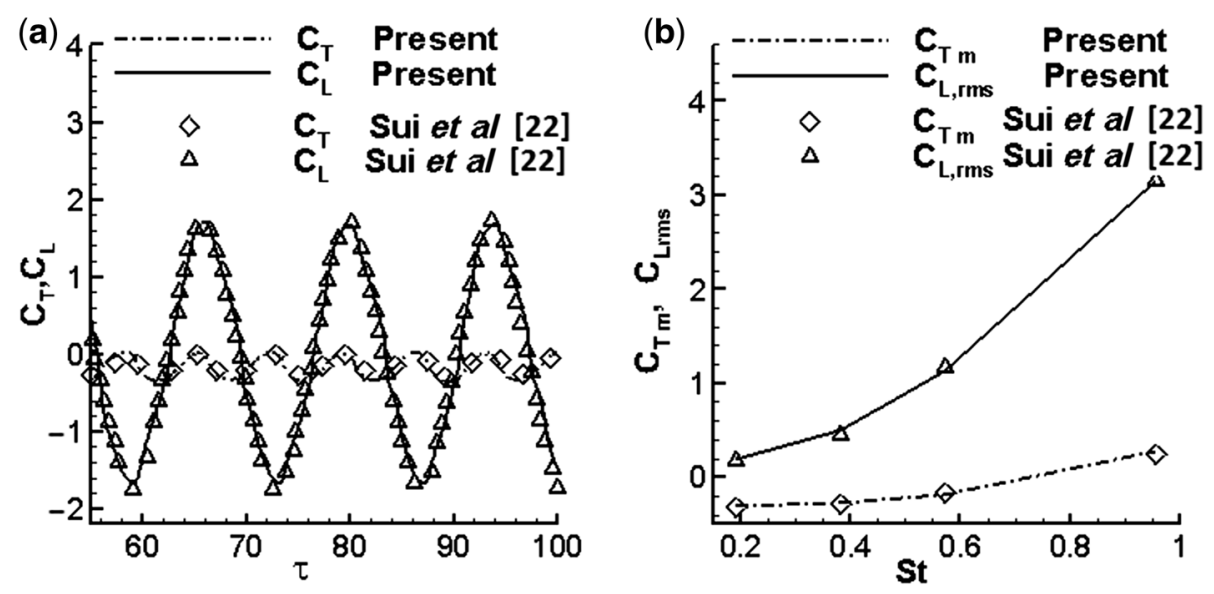

Figure 5. Comparison of present and published [22] results for flow across undulating NACA0012 hydrofoil, at $R e=400, A_{m a x}=0.2$ and $\lambda=1.0$. Variation of (a) instantaneous thrust and lateral force coefficients with time, at $\mathrm{St}=0.573$ and (b) mean value of thrust coefficient and rms value of lateral force coefficient.

The validation of the code, used in the present work, was presented in Shrivastava et al [21] - with an excellent agreement between the published experimental and numerical results. This was done extensively for various cases of linearly oscillating cylinder: one and two cylinders, unconfined and channel-confined flow, and streamwise and transversely oscillating cylinder and also for flow across a pitching hydrofoil. Further validation for the problem studied here is presented in figure 5. For the free-stream flow across an undulating NACA0012 hydrofoil, with amplitude varying as $A(X)=0.02-0.08 X+0.16 X^{2}$, the figure shows an excellent agreement of the present and the published [22] results at $R e=400, \lambda=1.0$ and $A_{\max }=0.2$. The agreement is shown for the instantaneous, mean and rms value of force coefficients.

\section{Results and discussion}

For the free-stream flow over an undulating NACA0012 hydrofoil, the effect of chordwise flexibility is studied by varying the wavelength of undulation $\lambda$ from 0.8 to 8.0 , at a constant Reynolds number $R e=4000$, Strouhal number $S t=0.4$ and maximum non-dimensional amplitude at the tail $A_{\max }=0.1$. The non-dimensional parameters selected in this study correspond to the swimming conditions of a real fish. Undulating motion is mostly found in slow moving swimmers with a wavelength of undulation less than the body length [23]. The smallest wavelength considered in this study $(\lambda=0.8)$ corresponds to many undulating fishes [23]. These types of fishes usually swim at a moderate Reynolds number with a non-dimensional frequency of $S t \approx 0.4$. The maximum non-dimensional amplitude of undulation $A_{\max }=0.1$ is observed in most of the fishes [23].
The results are presented below for scientifically exciting instantaneous flow patterns, and engineering-relevant engineering-parameters. The parameters considered here are the thrust coefficient $C_{T}$ the lateral force coefficient $C_{L}$ and the non-dimensional input power for undulation $P_{i n}$, given as follows:

$$
C_{T}=\frac{F_{T}}{1 / 2 \rho u_{\infty}^{2} c}, C_{L}=\frac{F_{L}}{1 / 2 \rho u_{\infty}^{2} c}, P_{i n}=\frac{\int_{S} f_{L} V_{b o d y} d S}{\rho u_{\infty}^{3} c}
$$

where $F_{T}$ is the thrust force per unit depth, $F_{L}$ is the lateral force per unit depth, $\rho$ is the density of the fluid, $f_{L}$ is the local lateral force per unit area on the surface and $V_{b o d y}$ is the undulating body velocity.

\subsection{Instantaneous flow patterns for undulating and pitching hydrofoils}

The temporal variation of the instantaneous flow patterns is presented in figures 6 and 7, for $A_{\max }=0.1, S t=0.4$ and $R e=4000$, at the smallest wavelength $(\lambda=0.8)$ and the largest wavelength $(\lambda=8.0)$ simulated here. The respective value of $\lambda$ corresponds to the undulating and pitching motion of hydrofoil. The temporal variations of the instantaneous flow patterns are presented at the same four increasing time instants, with a time interval of one-fourth of the time period of undulation between two consecutive flow patterns. The flow patterns correspond to an overlapped vorticity contours and velocity vectors in figure 6 , and pressure contours in figure 7 . For both undulating hydrofoil and pitching hydrofoil, the figures show that the ranges of values for the vorticity and velocity in the contour plots are the same (represented by a single colour bar), and different for the pressure contour. 


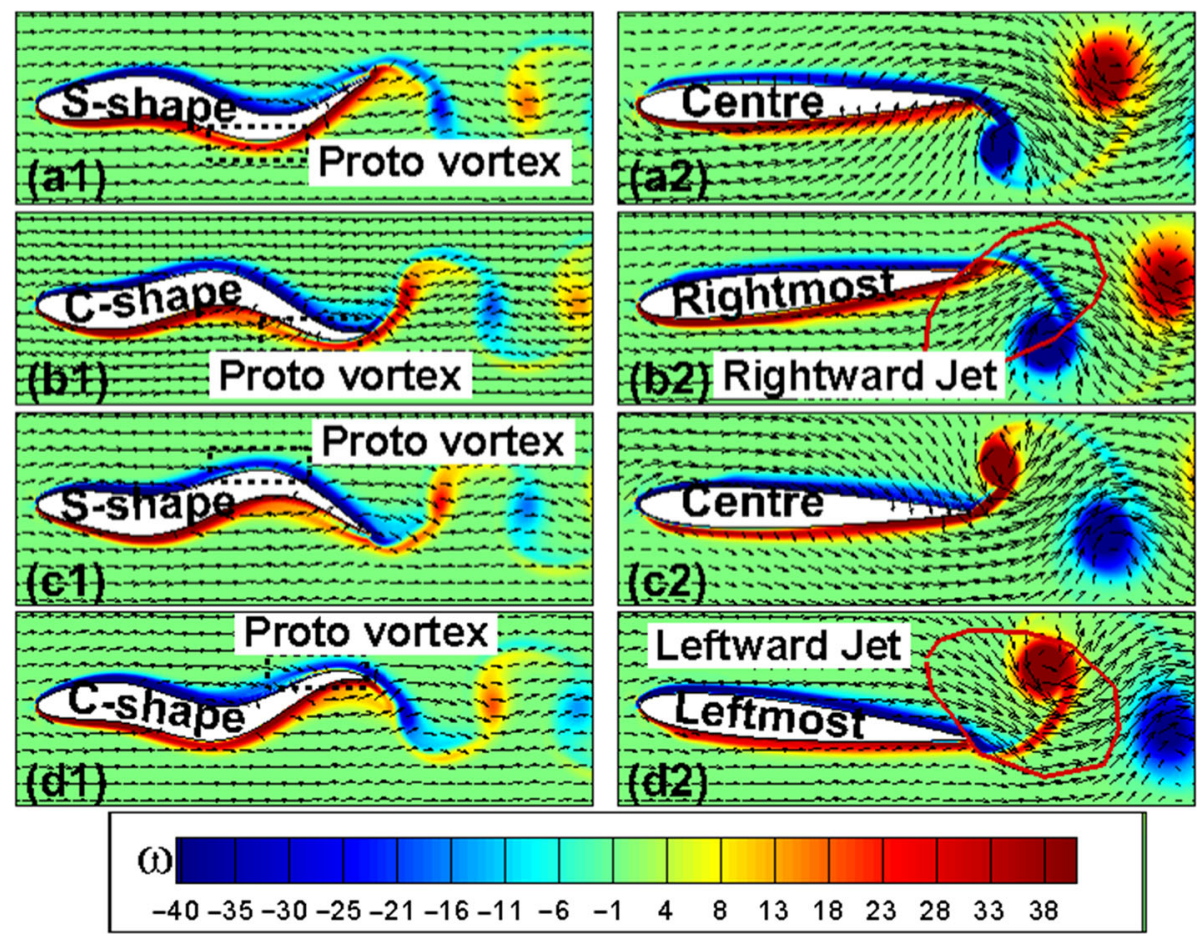

Figure 6. Temporal variation of instantaneous vorticity contours and velocity vectors, within one time period of (a1)-(d1) undulating (at $\lambda=0.8$ ) and (a2)-(d2) pitching (at $\lambda=8.0$ ) hydrofoil, at $A_{\max }=0.1, S t=0.4$ and $R e=4000$.
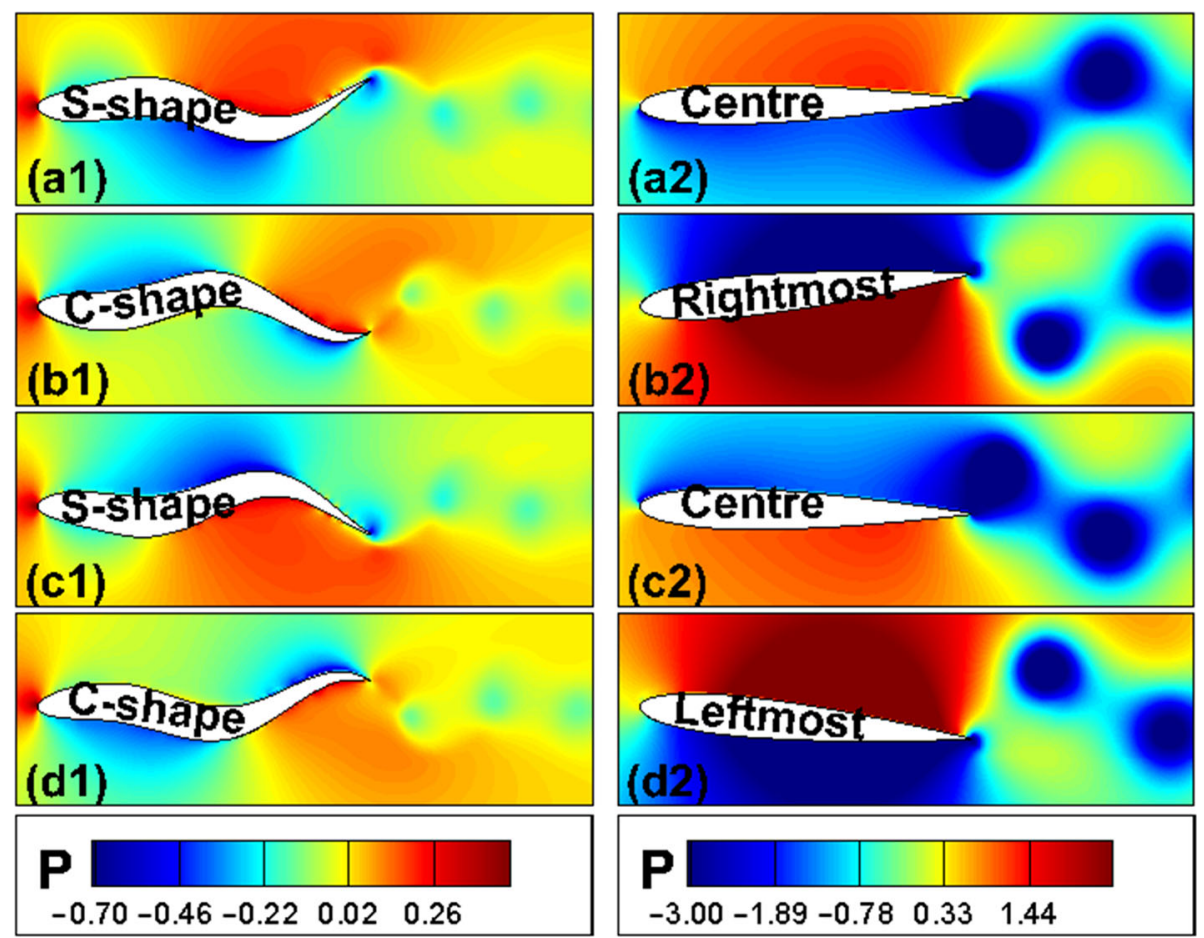

Figure 7. Temporal variation of instantaneous pressure contour, within one time period of (a1) $-(\mathbf{d} 1)$ undulating (at $\lambda=0.8)$ and (a2)(d2) pitching (at $\lambda=8.0$ ) hydrofoil, at $A_{\max }=0.1$, St $=0.4$ and $R e=4000$.

Figure 6a1-d1 shows that the fluid is pushed on one side (pressure side) and sucked on the other side (suction side), during the backwards passing of the wave travelling across the body of the foil. Therefore, the pressure-suction-based thrust generation mechanism [24] applies to the undulating hydrofoil at $\lambda=0.8$. This pressure and suction flow, 
together with increasing amplitude of undulation (from head to tail), increases the streamwise velocity of the fluid downstream of the foil, and a jet is ejected behind the hydrofoil. From the vorticity contour and velocity vector shown in figure $6 a 1-d 1$, it is clear that the pushing of the fluid leads to a semicircular flow of Clock-Wise (CW) sense on the right side and Counter-Clock-Wise (CCW) sense on the left side of the foil. The respective flow leads to the $\mathrm{CW} /$ negative and $\mathrm{CCW} /$ positive vortex on the respective side of the foil - called as primary vortices. Along with the negative primary vortex on the right side, a negative secondary vortex of much smaller size is shown (within a rectangular region) on the other (left) side in figure $6 \mathrm{a} 1$ and b1; similarly, figure $6 \mathrm{c} 1$ and $\mathrm{d} 1$ shows the $\mathrm{CCW} /$ positive primary and secondary vortex. The same signed primary and secondary vortices, on the two different sides, are due to the pushing and suction created by the travelling wave, respectively. These two vortices together resemble the potential part of a vortex, called as protovortex [6]. The proto-vortex formation can be clearly seen from the vorticity contours. As the wave travels backwards, the proto-vortex moves towards the tail and is shed off. The continuous pushing of the fluid distributes the thrust generation over the complete body.

For the pitching hydrofoil at $\lambda=8.0$, figure $6 \mathrm{a} 2-\mathrm{d} 2$ shows that formation of a starting and stopping vortex leads to the formation of a reverse von Karman vortex street. Figure $6 \mathrm{a} 2$ and $\mathrm{b} 2$ shows that when the foil moves from the central position towards extreme right position, the deceleration of the pitching motion of the foil results in deceleration of the CCW/positive vortex near the left surface called as the stopping vortex. The figure also shows an acceleration of the fluid near the tip of the left surface and the formation of the rightwards jet, shown by the encircled region in figure $6 \mathrm{~b} 2$. The resulting velocity difference across the tail-tip continues as the foil reverses the direction with an acceleration from the rightmost to the central position (figure $6 \mathrm{~b} 2$ and $\mathrm{c} 2$ ), resulting in the growth of the $\mathrm{CCW} /$ positive vortex at the tail-tip (figure $6 \mathrm{c} 2$ ) - called as the starting vortex. Similarly, figure $6 \mathrm{c} 2, \mathrm{~d} 2$ and a 2 shows the $\mathrm{CW} /$ negative stopping and starting vortex, and the leftwards jet, near the tail. The formation of the starting and stopping vortices leads to a vortex street, with the rightwards position of the CCW vortex (shed from the left surface) as compared with the CW vortex (shed from the right surface) - called as the reverse von Karman vortex street, seen in the figure for both undulating and pitching foils.

Comparing the vorticity contours for the pitching as compared with the undulating foil in figure $6 \mathrm{a} 1-\mathrm{d} 2$, it is interesting to note that the magnitude of vorticity is larger; thus, the strength of the reverse von Karman vortex street is stronger for the pitching foil. This results in the velocity vector plot, with a stronger jet flow behind the pitching as compared with the undulating hydrofoil.

Figure 7a1-d1 shows the pressure contours, at a timeinstant corresponding to " $\mathrm{S}$ " and "C"-like shape of the undulating hydrofoil, for $\lambda=0.8$. Note that the $\mathrm{S}$-like shape of the foil in figure $7 \mathrm{a}$ and c consists of one crust and one trough while the C-like shape consists of one crest in figure $7 \mathrm{~b}$ and one trough in figure $7 \mathrm{~d}$. It is interesting to note that the instantaneous " $S$ " and " $C$ "-like shape of the undulating foil leads to the minimum and maximum thrust coefficient, respectively, shown latter in figure 9a. Figure 7a1-d1 shows a larger pressure on one side of the hydrofoil where the fluid is pushed by the foil, and a smaller pressure is seen on the other side of the foil, called as pressure and suction side, respectively. The pressure and suction lead to a pressure difference between the anterior and posterior sections, which results in a net thrust force.The undulation of the hydrofoil results in momentum transfer to the surrounding fluid, in the streamwise as well as lateral directions. The maximum thrust generation corresponds to the maximum increase in the streamwise momentum. This is found when the foil takes a " $C$ " shape, shown in figures $7 \mathrm{~b} 1$ and $7 \mathrm{~d} 1$, where the area to push the fluid backwards is maximum [5]. The pressure distribution near the $\mathrm{C}$-shaped foil is such that it leads to the maximum pressure force in the streamwise direction, whereas, when the foil takes "S" shape, the pressure distribution in figures $7 \mathrm{a} 1$ and $\mathrm{c} 1$ is such that the component of the pressure force acting on the foil is larger in the lateral as compared with streamwise direction. The pushing area is almost horizontal, which reduces the momentum transfer in the streamwise direction and most of the momentum transfer is rather contributed to the lateral direction, thus it is found (presented later) to lead to the minimum thrust coefficient and maximum lateral force coefficient, respectively.

For the pitching hydrofoil at $\lambda=8.0$, the instantaneous pressure contours in figure $7 \mathrm{a} 2$ and $\mathrm{b} 2$ show that the pressure decreases near the right surface and increases near the left surface of the foil, and an opposite variation is seen in figure $7 \mathrm{~b} 2$ and $\mathrm{c} 2$. The former (latter) variation is due to the decelerated (accelerated) motion of the foil as it moves from the central (rightmost) to the rightmost (central) position. With further decelerated motion from the central to the leftmost position, followed by the accelerated motion towards the central position, a similar variation is seen on the left and right sides of the foil in figure $7 \mathrm{c} 2, \mathrm{~d} 2$ and $\mathrm{a} 2$. The pressure difference on the right and left surfaces results in a net pressure force acting in a direction slightly inclined to the horizontal axis. The component of this force in the streamwise direction is the pressure thrust force. For the undulating as compared with the pitching foil, the difference in the range of values of the pressure variation in figure 7 shows that the pressure force is much larger for the pitching hydrofoil.

For the undulating motion of the hydrofoil, figure 8 shows that the vorticity contours overlapped with the velocity vector $(\mathrm{a} 1-\mathrm{d} 1)$ and pressure contours $(\mathrm{a} 2-\mathrm{d} 2)$ at an intermediate wavelength $\lambda=1.2, R e=4000, S t=0.4$ and $A_{\max }=0.1$. The instantaneous flow field corresponds to maximum or minimum thrust generation conditions, shown 
marked inside the foil in the figure. The presence of protovortex is clearly seen in the vorticity contour, which shows that the thrust generation is due to the pressure-suction mechanism. From the velocity vector, it is clear that when the tail is almost at the extreme left (right) positions, a jet is formed towards the left (right) direction, which is almost similar to the jet flow in the pitching hydrofoil. The pressure contours shown in figure $8 \mathrm{a} 2-\mathrm{d} 2$ are similar to those in figure $7 \mathrm{a} 1-\mathrm{d} 1$ for the undulating hydrofoil at lower wavelength, such that the maximum thrust generation condition occurs when the area to push forward is maximum. Also, the tail of the foil is almost at the extreme left/right positions for the maximum thrust generation conditions. In this position, low and high pressure regions are formed on the posterior part of the foil, similar to the one observed in figure $7 \mathrm{a} 2-\mathrm{d} 2$ for the pitching foil. Hence, the thrust generation in the intermediate wavelength can be considered as the combination of pressure-suction mechanisms and the thrust generation due to the formation of alternate low and high pressure regions as found in the pitching motion.

\subsection{Analysis of thrust and lateral-force coefficients}

Within one time period of the periodic motion of undulating and pitching foil, figure 9 shows the temporal variation of thrust coefficient, lateral force coefficients and the position of the trailing edge of the hydrofoil $\left(Y_{\text {tail }}\right)$, for $A_{\max }=0.1, S t=0.4$ and $R e=4000$. The figure also shows four symbols, with labels a-d, corresponding to the results at the four time instants for which the flow patterns are shown in the parts a-d of figures 6 and 7.

For the undulating hydrofoil at $\lambda=0.8$, at the time instants corresponding to the " $\mathrm{C}$ " shape of the foil (figures 6 and $7 b$ and d), the symbols " $b$ " and "d" in figure 9a show that the thrust-coefficient is maximum, lateral force coefficient is almost zero, and the tail is slightly behind the extreme left (or right) position. Thus, thrust is generated in the undulation motion with minimum power loss in the lateral direction, and almost all the momentum transferred from the foil to the fluid is converted into the thrust force. Furthermore, corresponding to the " $\mathrm{S}$ " shape of the foil in figures 6 and $7(\mathrm{a}$ and $\mathrm{c})$, figure 9a shows that the thrust coefficient is minimum, the lateral force coefficient is maximum and the tail is slightly ahead of the extreme left (or right) position.

For the pitching hydrofoil at $\lambda=8.0$, the symbols " $\mathrm{b}$ " and "d" in figure 9b show that the time instant for the maximum of the thrust coefficient almost corresponds to the maximum of the lateral force coefficient as well as the extreme left (or right) position of the foil. The maximum deceleration at the extreme position results in the largest pressure force on the surface of the foil. Furthermore, the symbols "a" and "c" in the figure show that the minimum of the thrust generation almost corresponds to zero lateral force coefficient and the central position of the foil, where the acceleration (or deceleration) of the foil is zero. Note from the figure that the lateral force coefficient is much larger than the thrust coefficient. Moreover, since the

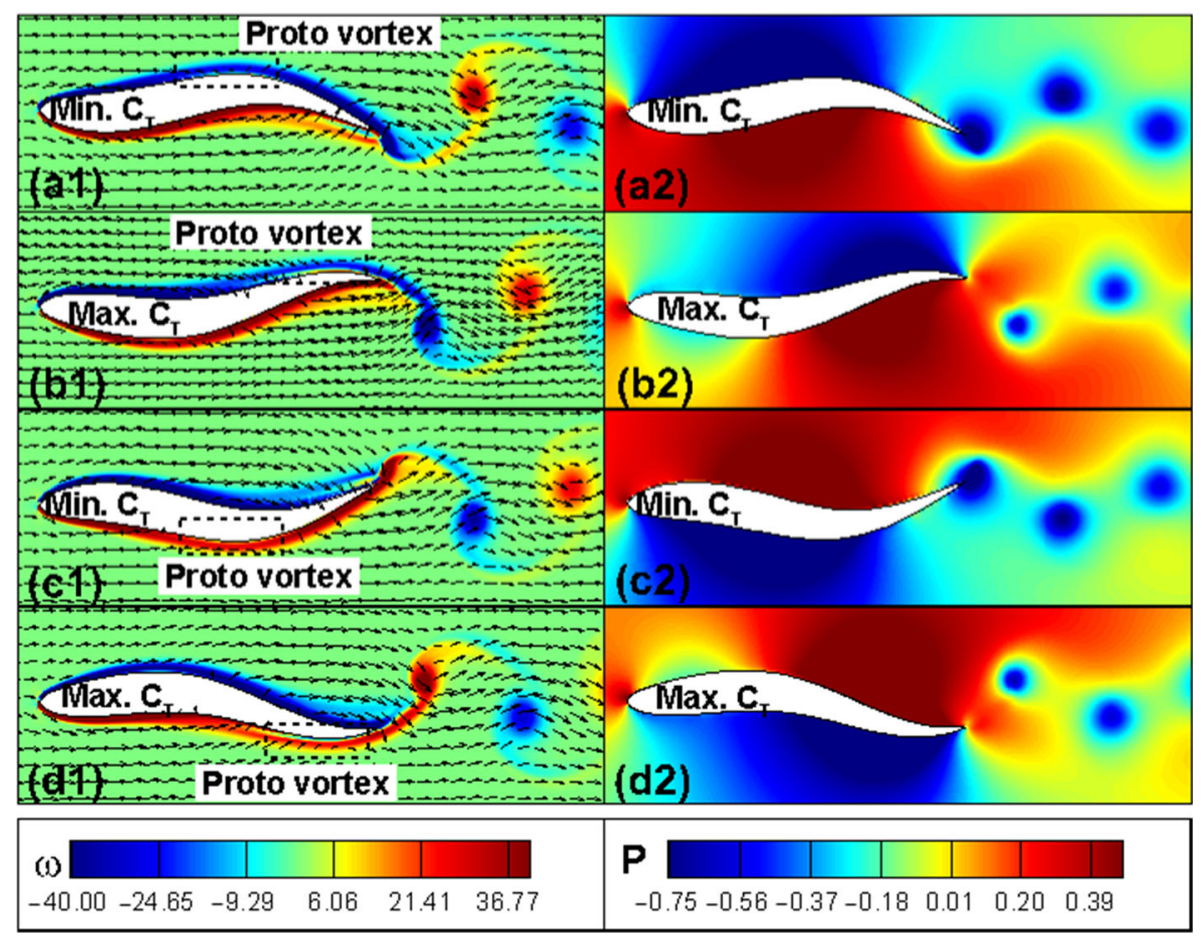

Figure 8. Temporal variation of instantaneous (a1)-(d1) vorticity contours with velocity vectors and (a2)-(d2) pressure contours within one time period of undulating motion at $\lambda=1.2, A_{\max }=0.1, S t=0.4$ and $R e=4000$. 


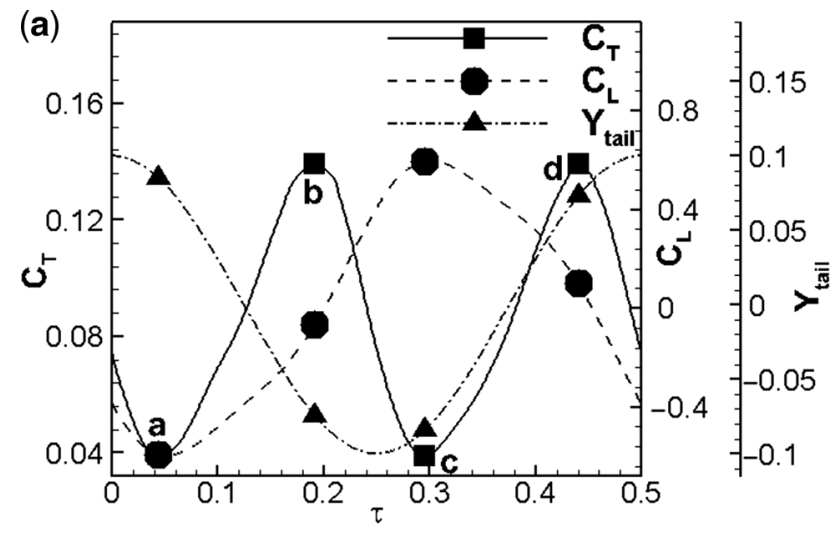

(b)

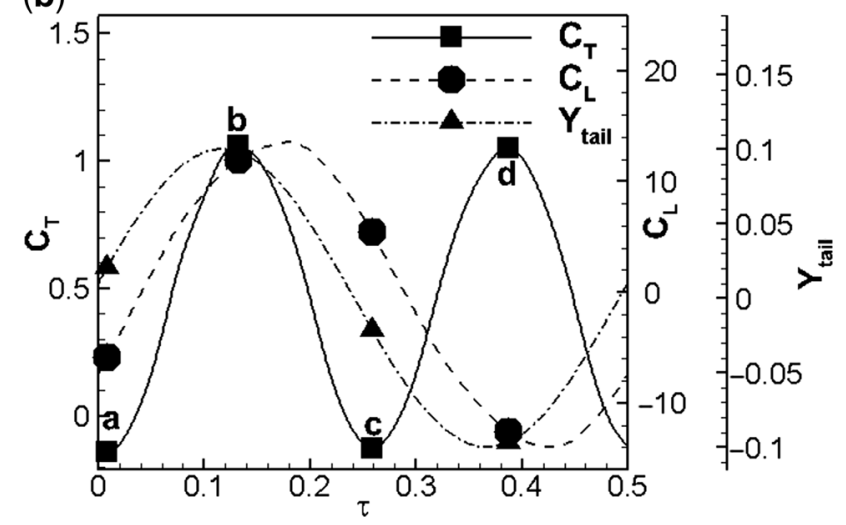

Figure 9. Temporal variation of thrust coefficient, lateral force coefficient and the position of the trailing edge of the hydrofoil $\left(Y_{\text {tail }}\right)$, within one time period of (a) undulating (at $\lambda=0.8$ ) and (b) pitching (at $\lambda=8.0$ ) hydrofoil, at $A_{\max }=0.1, S t=0.4$ and $R e=4000$. The symbols " $a-d$ " correspond to the time instants at which instantaneous flow patterns are shown in figures 6 and 7 .

maximum of both the coefficients occurs at almost the same time instant, only a small part of the energy spend on the pitching motion of the hydrofoil is converted into thrust coefficient. Also, the ratio of maximum thrust to maximum lateral force coefficient is larger for the undulating as compared with pitching foil.

From the temporal variation of lateral-force and thrust coefficient at various wavelengths, a phase difference is found between the maximum lateral-force coefficient and the maximum thrust coefficient. This is shown in figure 10, with an almost asymptotic decrease in the phase difference with increasing wavelength; note that the asymptotic value almost corresponds to $0^{o}$, i.e., in-phase. Thus, the thrust generation is by the pressure-suction mechanism at the smaller wavelength, and a different mechanism (discussed earlier for pitching motion) at larger wavelength; at an intermediate wavelength, the thrust generation mechanism corresponds to the combination of the two mechanisms.

With increasing wavelength, the variations of the mean (time-averaged) thrust coefficient $\left(C_{T m}\right)$, the ratio of maximum thrust coefficient $\left(C_{T \max }\right)$ to maximum lateral force coefficient $\left(C_{\text {Lmax }}\right)$ and input power $\left(P_{\text {in }}\right)$ are shown in figure 11, at $A_{\max }=0.1, S t=0.4$ and $R e=4000$. With increasing $\lambda$, figure 11a shows that $C_{T m}$ increases monotonically and asymptotes to the value corresponding to the pure pitching motion. Thrust generation in pitching (large wavelength) motion is by the formation of low and high pressure regions, discussed earlier. It is worth noting from the figure that this mechanism at larger $\lambda$ generates higher thrust compared with the pressure suction mechanism at lower $\lambda$. Reduction in the wavelength, with a superposition of undulation on the pitching motion, is equivalent to adding chordwise flexibility to the foil. The flexibility results in thrust generation due to pushing of the fluid [25]. For the lowest wavelength, thrust generation is entirely due to the pressure-suction mechanism. Furthermore, for the lower range of value of $\lambda$, the figure shows a linear variation in $C_{T m}$. This is because, for the pressure-suction mechanism, the thrust is strongly dependent on the pushing of the fluid by the wave velocity $(\lambda f)$.

With increasing $\lambda$, figure $11 \mathrm{~b}$ shows an asymptotic variation - with decrease in the ratio of $C_{T \max }$ to $C_{L \max }$ and increase in the input power $P_{i n}$, and the asymptotic value corresponding to the value of the pure pitching motion. The lowest wavelength leading to the largest value of the ratio indicates that the undulating motion, with the pressuresuction mechanism, leads to a larger fraction of the momentum transfer in the streamwise direction as compared with that for the larger wavelength corresponding to the pitching motion. This results in the larger power requirement for lateral undulatory motion at higher $\lambda$. With increasing $\lambda$, there is a much sharper increase in $C_{L \max }$ as compared with $C_{\text {Tmax }}$; however, both the coefficient asymptotes to a value corresponding to the pure pitching motion.

\section{Conclusion}

A level-set based immersed boundary method is used to study the free-stream flow across an undulating NACA0012 hydrofoil. The study on the effect of chordwise flexibility of the hydrofoil shows that the hydrodynamics results for undulating motion asymptote that for the pitching motion, with an increase in the wavelength of undulation. The mechanism of thrust generation due to undulating motion (at smaller wavelength) is found to be different from the mechanism for the pitching motion (at larger wavelength). For the undulating motion, the fluid is pushed on one side (pressure side) and sucked on the other side (suction side). The continuous pushing and suction are found to generate thrust, and the maximum thrust coefficient and minimum lateral force coefficient are found to occur almost at the same instant. However, for the pitching motion, the thrust generation is due to the formation of alternate low and high pressure regions on the surfaces followed by the 


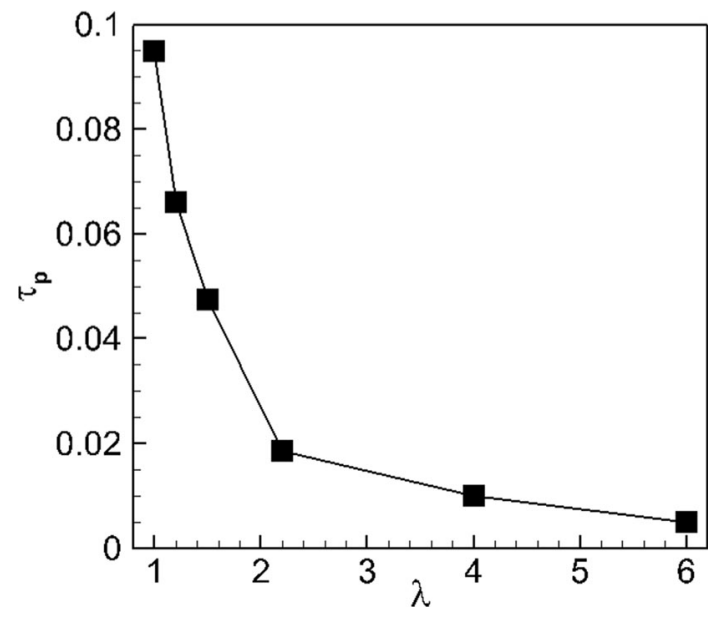

Figure 10. Variation of phase difference between maximum lateral-force coefficient and maximum thrust coefficient (obtained from their periodic temporal variations), with increasing wavelength, at $A_{\max }=0.1, S t=0.4$ and $R e=4000$.
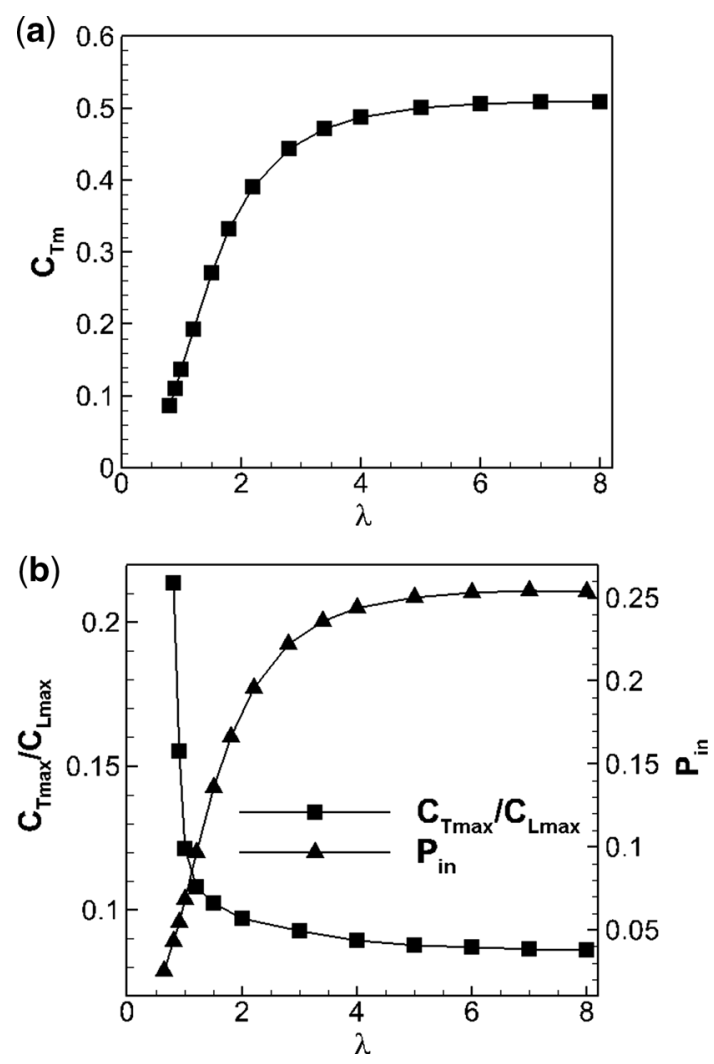

Figure 11. Variation of (a) mean thrust coefficient and (b) ratio of maximum thrust coefficient $\left(C_{T \max }\right)$ to maximum lateral force coefficient $\left(C_{\text {Lmax }}\right)$ and input power $\left(P_{\text {in }}\right)$ with increasing wavelength, at $A_{\max }=0.1, \operatorname{Re}=4000$ and $S t=0.4$.

accelerating and decelerating motion of the hydrofoil with a larger fraction of momentum transfer in the lateral direction. Furthermore, the maximum thrust coefficient and maximum lateral force coefficient are found to occur almost at the same instant. The ratio of maximum thrust coefficient to maximum lateral force coefficient is found to be higher for the undulating motion with smaller wavelength, and the mean thrust coefficient is found to be larger for the large wavelength undulating motion. Thus, it can be concluded that undulating motion with smaller wavelength can be preferred for smaller thrust force applications with minimum momentum loss in the lateral direction, whereas undulating motion with larger wavelength (corresponding to pitching) can be used for applications where larger thrust generation is required.

\section{References}

[1] Breder C M 1926 The locomotion of fishes. Zoologica 4: 159-256

[2] Sfakiotakis M, Lane D M and Davies J B C 1999 Review of fish swimming modes for aquatic locomotion. IEEE $J$. Ocean. Eng. 24(2): 237-252

[3] Magnuson J J 1979 Locomotion by scombrid fishes: hydromechanics, morphology, and behavior. In: Hoar W S and Randall D J (Eds.) Fish physiology. Cambridge: Academic Press, vol. 4, pp. 239-313

[4] Lighthill M J 1960 Note on the swimming of slender fish. $J$. Fluid Mech. 9(02): 305-317

[5] Liu H, Wassersug R and Kawachi K 1996 A computational fluid dynamics study of tadpole swimming. J. Exp. Biol. 199(6): 1245-1260

[6] Müller U K, Smit J, Stamhuis E J and Videler J J 2001 How the body contributes to the wake in undulatory fish swimming. J. Exp. Biol. 204(16): 2751-2762

[7] Tytell E D and Lauder G V 2004 The hydrodynamics of eel swimming I. Wake structure. J. Exp. Biol. 207(11): 1825-1841

[8] Jian D, Shao X and Ren A 2006 Numerical study on propulsive performance of fish-like swimming foils. $J$. Hydrodyn. 18(6): 681-687

[9] Kern S and Koumoutsakos P 2006 Simulations of optimized anguilliform swimming. J. Exp. Biol. 209(24): 4841-4857

[10] Borazjani I and Sotiropoulos F 2008 Numerical investigation of the hydrodynamics of carangiform swimming in the transitional and inertial flow regimes. J. Exp. Biol. 211(10): $1541-1558$

[11] Borazjani I and Sotiropoulos F 2009 Numerical investigation of the hydrodynamics of anguilliform swimming in the transitional and inertial flow regimes. J. Exp. Biol. 212(4): 576-592

[12] Borazjani I and Sotiropoulos F 2010 On the role of form and kinematics on the hydrodynamics of self-propelled body/caudal fin swimming. J. Exp. Biol. 213(1): 89-107

[13] Koochesfahani M M 1989 Vortical patterns in the wake of an oscillating airfoil. AIAA J. 27(9): 1200-1205

[14] Pedro G, Suleman A and Djilali N 2003 A numerical study of the propulsive efficiency of a flapping hydrofoil. Int. J. Numer. Methods Fluids 42(5): 493-526

[15] Shinde S Y and Arakeri J H 2013 Jet meandering by a foil pitching in quiescent fluid. Phys. Fluids 25(4): 041701 
[16] Triantafyllou G S, Triantafyllou M S and Grosenbaugh M A 1993 Optimal thrust development in oscillating foils with application to fish propulsion. J. Fluids Struct. 7(2): 205-224

[17] Anderson J M, Streitlien K, Barrett D S and Triantafyllou M S 1998 Oscillating foils of high propulsive efficiency. $J$. Fluid Mech. 360: 41-72

[18] Gopalkrishnan R, Triantafyllou M S, Triantafyllou G S and Barrett D 1994 Active vorticity control in a shear flow using a flapping foil. J. Fluid Mech. 274: 1-21

[19] Guglielmini L and Blondeaux P 2004 Propulsive efficiency of oscillating foils. Eur. J. Mech. B Fluids 23(2): 255-278

[20] Sohankar A, Norberg C and Davidson 1998 Low-Reynoldsnumber flow around a square cylinder at incidence: study of blockage, onset of vortex shedding and outlet boundary condition. Int. J. Numer. Methods Fluids 26(1): 39-56.

[21] Shrivastava M, Agrawal A and Sharma A 2013 A novel level set-based immersed boundary method for CFD simulation of moving-boundary problems. Numer. Heat Transfer B 63(4): 304-326

[22] Sui Y, Chew Y T, Roy P and Low H T 2007 A hybrid immersed-boundary and multi-block lattice Boltzmann method for simulating fluid and moving-boundaries interactions. Int. J. Numer. Methods Fluids 53(11): 1727-1754

[23] Videler J J and Wardle C S 1991 Fish swimming stride by stride: Speed limits and endurance. Rev. Fish Biol. Fish. 1(1): 23-40

[24] Müller U K, van den Heuvel B L E, Stamhuis E J and Videler J J 1997 Fish foot prints: morphology and energetics of the wake behind a continuously swimming mullet (chelon labrosus Risso). J. Exp. Biol. 201: 2893-2906

[25] Marais C, Thiria B, Wesfreid J E and Godoy-Diana R 2012 Stabilizing effect of flexibility in the wake of a flapping foil. J. Fluid Mech. 710: 659-669 